\title{
Gastric Ulcers with Cytomegalovirus Infection in an Immunocompetent Patient
}

\author{
Tae Oh Kim, Ki-Nam Shim, Sang Yoon Kim, Ji Young Lim, A Reum Choe, Chung Hyun Tae, Chang Mo Moon, Seong-Eun Kim, \\ Hye-Kyung Jung, Sung-Ae Jung \\ Department of Internal Medicine, Ewha Medical Research Institute, Ewha Womans University College of Medicine, Seoul, Korea
}

\begin{abstract}
Cases of cytomegalovirus (CMV) infection are rare, and this infection is commonly asymptomatic in immunocompetent patients. Here, we report a pathologically proven case of CMV infection in gastric ulcers in an immunocompetent patient. A 35-year-old man visited our hospital for a surveillance esophagogastroduodenoscopy. Except fatigue, he had no other symptoms. Laboratory data showed that his white cell count was $9.28 \times 10^{3} / \mu \mathrm{L}$ with $60.1 \%$ lymphocytes. However, aspartate transaminase and alanine aminotransferase levels were elevated. Esophagogastroduodenoscopy revealed multiple gastric ulcers that were healing. Two endoscopic biopsies were performed to obtain specimens at the ulcer base. Histological examination and immunohistochemistry confirmed CMV infection; subsequently, we decided that the best option was observation without medication. He revisited a month later, and the ulcers had disappeared. (Korean J Helicobacter Up Gastrointest Res 2019;19:277-281)
\end{abstract}

Key Words: Cytomegalovirus; Immunocompetence, Stomach ulcer

\section{INTRODUCTION}

Human disease occurring due to cytomegalovirus (CMV) varies and is largely host-dependent. CMV infection in immunocompromised patients leads to substantial morbidity and mortality, especially among those who are transplanted and infected with human immunodeficiency virus (HIV). ${ }^{1}$ However, it is usually asymptomatic, but can cause symptoms like mononucleosis syndrome, in immunocompetent patients. ${ }^{2}$ Generally, CMV infections involve many organs, such as the lungs, liver, central nervous system, and gastrointestinal tract, mostly common in the stomach and colon. ${ }^{1}$ Gastrointestinal CMV infection is rare in hosts with normal immune system. ${ }^{3}$ Majority of reported cases are CMV colitis. ${ }^{4}$ This report described that the presence of abnormal mucosal surfaces prior to $\mathrm{CMV}$ infection could increase the risk of its occurrence. Only a few cases of cytomegalic cells in gastrointestinal tract ulcers in normal hosts have been reported. ${ }^{5} \mathrm{CMV}$ infection in the stomach generally causes hypertrophic and erosive gastritis with superficial and deep

Received: July 15, 2019 Revised: August 17, 2019 Accepted: August 17, 2019 Corresponding author: Ki-Nam Shim

Department of Internal Medicine, Ewha Medical Research Institute, Ewha Womans University College of Medicine, 260 Gonghang-daero, Gangseo-gu, Seoul 07804, Korea Tel: +82-2-6986-1622, Fax: +82-2-2650-5312, E-mail: shimkn@ewha.ac.kr ulceration. ${ }^{6}$ This is a case report of $\mathrm{CMV}$ infection in gastric ulcers confirmed through biopsy in an immunocompetent patient.

\section{CASE REPORT}

A 35-year-old man visited Ewha Womans University Medical Center for a surveillance esophagogastroduodenoscopy (EGD). He had no symptoms of epigastric pain, hematemesis, melena, and hematochezia. He was afebrile and did not have any medical history. His medical history was nonspecific, especially gastrointestinal disease. He was a never-smoker with history of recent social alcohol ingestion.

Laboratory data showed hemoglobin level of $15.2 \mathrm{~g} / \mathrm{dL}$ and hematocrit of $45.9 \%$. His white blood cell count was $9.28 \times 10^{3}$ with $60.1 \%$ lymphocytes (normal $20.5 \sim 51.1 \%$ ). Electrolyte, creatinine, ALP, and bilirubin levels were within normal limits; however, AST and ALT were elevated, i.e., 78 (normal 0 40) IU/L and 123 (normal 0 40) IU/L, respectively. Chest X-ray was normal.

EGD showed the healing status of multiple ulcers at the angle, antrum, and lesser curvature of the lower body (Fig. 1). Two endoscopic biopsies were obtained from the ulcers at the angle and lesser curvature of the antrum. Histologic examination confirmed chronic active gastritis

Copyright $\odot 2019$ Korean College of Helicobacter and Upper Gastrointestinal Research

(a) The Korean Journal of Helicobacter and Upper Gastrointestinal Research is an Open-Access Journal. All articles are distributed under the terms of the Creative Commons Attribution Non-Commercial License (http:// creativecommons.org/licenses/by-nc/4.0) which permits unrestricted non-commercial use, distribution, and reproduction in any medium, provided the original work is properly cited. 
with dense lymphoplasmacytic infiltration. Some intranuclear inclusions were suspicious of viral infections like CMV (Fig. 2A). Immunohistochemistry revealed CMV-positive results, with $\mathrm{CD} 20$ positive in some infiltrating $\mathrm{B}$ cells (Fig. 2B). Giemsa staining displayed no identifiable Helicobacter pylori (H. pylori). The patient was diagnosed with CMV-associated gastric ulcer, and then, a decision was made to perform observation only without medication, because the patient complained no symptoms, and shallow ulcers were already healing. He returned for a follow-up check-up a month later, and EGD was performed, which showed that the ulcers had been cleared (Fig. 3), and random biopsies from the gastric mucosa

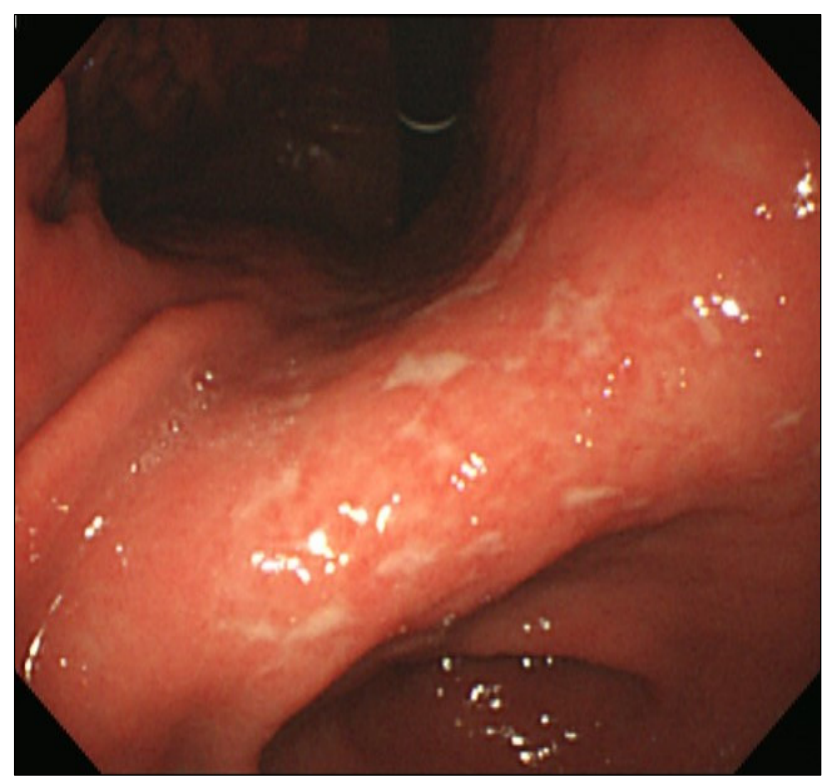

Fig. 1. Endoscopic findings of multiple healing ulcers at the angle, antrum, and lesser curvature of the lower body. showed active H. pylori gastritis. Therefore, H. pylori eradication therapy has been initiated.

\section{DISCUSSION}

Herein, we report a case of pathologically proven CMV gastric ulceration in a young healthy immunocompetent $\mathrm{pa}^{-}$ tient who had a good prognosis without antiviral treatment. CMV infection has been known to commonly occur in immunodeficiency patients, such as those with HIV infection, who underwent solid organ or stem cell transplantation, and those undergoing chemo- or radiotherapy. ${ }^{1-3}$ However, in the last decades, CMV disease in immunocompetent patients

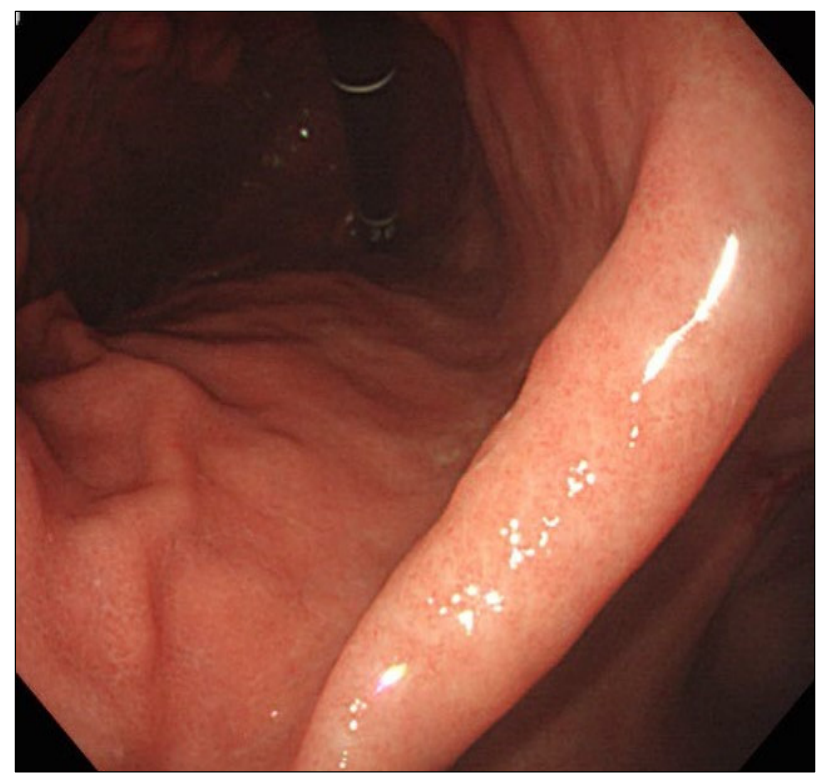

Fig. 3. Endoscopic findings showing disappearance of the ulcers a month later.
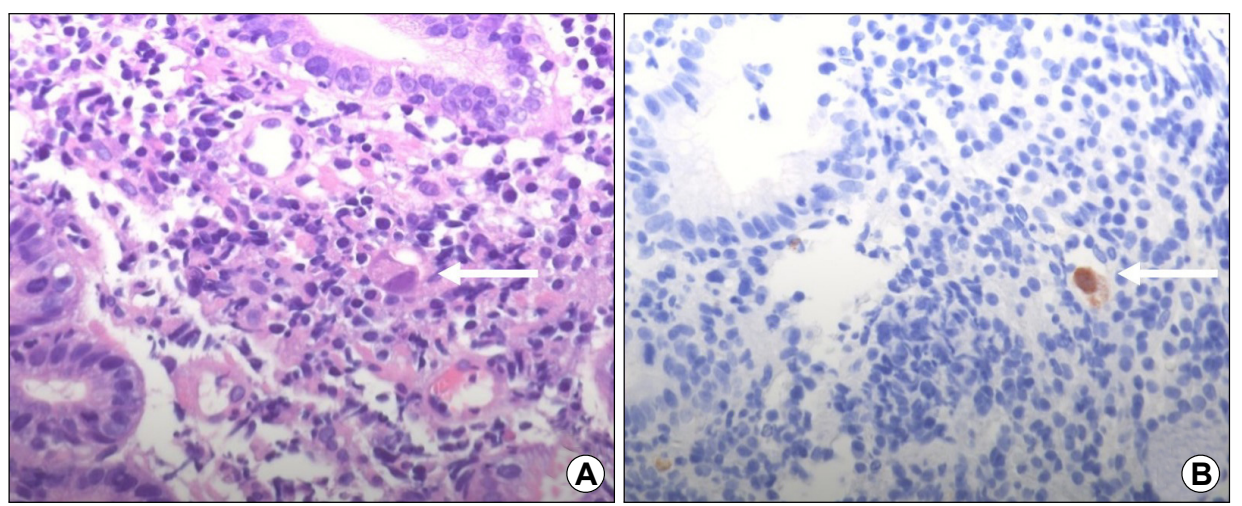

Fig. 2. Pathologic findings showing (A) owl's eye cells with enlarged nuclei and peripheral halos in the gastric epithelial cells (white arrow, H\&E, ×400) and (B) positivity for cytomegalovirus immunostaining (white arrow, immunohistochemical staining, $\times 400$ ). 
has been increasing, involving the upper gastrointestinal tract. ${ }^{4}$ The clinical presentation of upper gastrointestinal CMV disease widely varies and depends on its location or severity in immunocompetent patients. ${ }^{5}$ Epigastric pain, gastrointestinal bleeding, and nausea/vomiting were the most frequent complaints in patients with upper gastrointestinal CMV disease. ${ }^{6-8}$ Moreover, approximately $7 \%$ of patients with upper gastrointestinal CMV disease may have an asymptomatic presentation such as in our case. ${ }^{5}$

In a domestic report on CMV infection, the majority of immunocompetent patients were elderly, and many patients had accompanying diseases such as hemodialysis.? There are many studies and case reports on the association of CMV infection with autoimmune diseases. ${ }^{10}$ Recently, there is increasing evidence that CMV is associated with many malignancies. ${ }^{11}$ Therefore, there are important considerations when treating gastric ulcer patients with CMV co-infection. A thorough history taking of patients should be undertaken. Underlying disorders, infections, hidden malignancy and autoimmune disease that can reduce the patient's immunity should be identified. The test should include HIV antibody test, white blood cell, absolute neutrophil count, platelets count, peripheral blood smear and liver enzyme. In young patients with normal immunity, the tests mentioned above are sufficient. In addition, immunoglobulin levels, $\mathrm{T}$ cell counts, and complement tests are required for older patients, HIV-infected patients, hemodialysis patients, patients with rheumatic diseases, and patients undergoing chemotherapy or radiation therapy. ${ }^{10}$

Immunocompetent patients are defined as having no congenital immunodeficiency, HIV infection, no transplantation, no prior chemotherapy, or no immunosuppressive (including corticosteroid) use. This is a report of CMV infection in immunocompetent patients. He had no recent history of travel. He had no history of upper respiratory infection or fever, suggestive of viral infection, and no history of chemotherapy or radiation therapy. The aforementioned patient was healthy. He did not receive a blood transfusion for life, and had never taken steroids or drugs. He was not a risk group for acquired immunodeficiency syndrome. The patient was confirmed to have normal immunity by serial examination of complete blood count including absolute neutrophil count and peripheral blood smear.

The endoscopic appearance of upper gastrointestinal CMV diseases also varies and is nonspecific, ranging from normal feature to deep ulcer with perforation or massive bleeding. ${ }^{12}$ Erosions and ulcerations tend progress into multiple lesions in endoscopy. CMV disease is difficult to diagnose due to a wide array of potential clinical manifestations and endoscopic findings. Delayed diagnosis of CMV disease in immunocompetent adults can potentially result in numerous adverse outcomes. Delayed initiation of targeted therapy leads to increased morbidity and mortality as a result of disease progression.

CMV infection in immunocompromised patients can be very difficult to treat. The fact that ulcers and CMV inclusions resolved without specific antiviral treatment gives further support to the belief that the patient's immune system is normal. The relationship of symptomatic ulcer disease with CMV inclusion findings in mucosal biopsies of ulcers remains unclear. ${ }^{12}$ Whether CMV is a primary cause of gastrointestinal lesions or whether CMV colonizes preexisting lesions remain to be elucidated. ${ }^{13}$ Some experts view that mucosal injury due to another cause may be followed by CMV infection in the granulation tissue, with then may result in further injury and perforation. ${ }^{14}$ Others suggest that CMV plays an important role in the pathogenesis of localized gastrointestinal diseases. Furthermore, CMV is believed to may have play a role in the pathogenesis of acute gastrointestinal ulcers. ${ }^{12}$

In immunodeficient or immunosuppressed hosts, antiviral treatment for $\mathrm{CMV}$ disease in the gastrointestinal tract is recommended with intravenous ganciclovir or valganciclovir for 2 3 weeks. ${ }^{13}$ However, CMV infection has been considered self-limiting, and not all patients receive antiviral therapy. ${ }^{14,15}$ The role of antiviral therapy in immunocompetent patients remains controversial. Several studies reported that advanced age is a risk factor for CMV disease in immunocompetent hosts, in line with immune senescence. ${ }^{16,17}$ In addition, critical conditions or $\mathrm{co}^{-}$ morbidities can play a role in patients without immunodeficiency, causing significant impairment of the host's immune response. ${ }^{18,19}$ Therefore, an antiviral treatment should be considered in a case-by-case basis in immunocompetent patients, being often reserved for severe 
CMV disease, old age, and critical illness or comorbidities. In the absence of risk factors, antiviral treatment appears to have little benefit, because most untreated patients have good evolution without complications. Depending on the degree of gastric ulceration and these symptoms, we should consider the variety of treatment methods. As with the patient in this case, most immunocompetent patients can expect improvement only by observation. If $\mathrm{CMV}$ infected patients are treated with immunosuppressants, including corticosteroids, or if they are being treated with radiation chemotherapy, their cessation improves the lesion. If mononucleosis symptoms such as fever, epigastric pain, nausea, and vomiting are seen, supportive care such as fluid therapy and H2-receptor antagonist will be helpful. Antiviral and proton-pump inhibitor (PPI) treatments may be necessary because of the risk of perforation when the patient shows gastric ulcer on deep layer.'

In this patient, administration of a PPI for acid suppression would have been an option to heal the ulcers without concomitant administration of an antiviral agent such as ganciclovir. ${ }^{20}$ The gastric ulcers were completely healed within 4 weeks, and this rate was not considered to be delayed compared to $H$. pylori related ulcers. In this case, complete healing ulcers were confirmed within 4 weeks without any medication, including PPI treatment. Therefore, at this case, initial gastric ulcers were more related with CMV infection. In conclusion, we present a case of CMV gastric ulcers in a young healthy patient with good prognosis without antiviral treatment.

\section{CONFLICT OF INTEREST}

No potential conflict of interest relevant to this article was reported.

\section{ORCID}

$\begin{array}{ll}\text { Tae Oh Kim } & \text { (D https://orcid.org/0000-0002-6369-5618 } \\ \text { Ki-Nam Shim } & \text { (D) https://orcid.org/0000-0003-4004-6292 } \\ \text { Sang Yoon Kim } & \text { (D) https://orcid.org/0000-0002-1155-1495 } \\ \text { Ji Young Lim } & \text { (D) https://orcid.org/0000-0002-3252-9035 } \\ \text { A Reum Choe } & \text { (D) https://orcid.org/0000-0002-2552-7066 } \\ \text { Chung Hyun Tae } & \text { (D https://orcid.org/0000-0002-0764-7793 }\end{array}$

Chang Mo Moon (D) https://orcid.org/0000-0003-2550-913X Seong-Eun Kim (D) https://orcid.org/0000-0002-6310-5366 Hye-Kyung Jung (D) https://orcid.org/0000-0002-6653-5214 Sung-Ae Jung (D) https://orcid.org/0000-0001-7224-2867

\section{REFERENCES}

1. Gianella S, Letendre S. Cytomegalovirus and HIV: a dangerous pas de deux. J Infect Dis 2016;214 Suppl 2:S67-S74.

2. Maertens J, Lyon S. Current and future options for cytomegalovirus reactivation in hematopoietic cell transplantation patients. Future Microbiol 2017;12:839-842.

3. Goerig N, Semrau S, Frey B, et al. Clinically significant CMV (re)activation during or after radiotherapy/chemotherapy of the brain : correlation with neurological deterioration and improvement upon antiviral treatment. Strahlenther Onkol 2016;192:489-497.

4. Gravito-Soares E, Almeida N. Cytomegalovirus disease of the upper gastrointestinal tract: an emerging infection in immunocompetent hosts. GE Port J Gastroenterol 2017;24: 259-261.

5. Marques S, Carmo J, Pinto D, Bispo M, Ramos S, Chagas C. Cytomegalovirus disease of the upper gastrointestinal tract: a 10-year retrospective study. GE Port J Gastroenterol 2017; 24:262-268.

6. You DM, Johnson MD. Cytomegalovirus infection and the gastrointestinal tract. Curr Gastroenterol Rep 2012;14:334-342.

7. Galiatsatos P, Shrier I, Lamoureux E, Szilagyi A. Meta-analysis of outcome of cytomegalovirus colitis in immunocompetent hosts. Dig Dis Sci 2005;50;609-616.

8. Nasa M, Sharma Z, Sud R, Lipi L. Cytomegalovirus infection of gastrointestinal tract. Community Acquir Infect 2016;3:4-9.

9. Kim SW, Cha YJ, Kim MH, et al. Cytomegalovirus colitis with colon perforation and lower gastrointestinal bleeding in a immunocompetent patient. Ewha Med J 2014;37;105-108.

10. Halenius A, Hengel H. Human cytomegalovirus and autoimmune disease. Biomed Res Int 2014;2014:472978.

11. Zhang L, Guo G, Xu J, et al. Human cytomegalovirus detection in gastric cancer and its possible association with lymphatic metastasis. Diagn Microbiol Infect Dis 2017;88;62-68.

12. Iwamuro M, Kondo E, Tanaka T, et al. Endoscopic manifestations and clinical characteristics of cytomegalovirus infection in the upper gastrointestinal tract. Acta Med Okayama 2017;71: 97-104.

13. Hodowanec AC, Pikis A, Komatsu TE, et al. Treatment and prevention of CMV disease in transplant recipients: current knowledge and future perspectives. J Clin Pharmacol 2019;59: 784-798.

14. Fyock C, Gaitanis M, Gao J, Resnick M, Shah S. Gastrointestinal CMV in an elderly, immunocompetent patient. R I Med J (2013) 2014;97:53-56.

15. Kastenbauer U, Ließ H, Kremer M. CMV-associated gastric ul- 
cer in an immunocompetent male patient. Internist (Berl) 2016;57:724-727.

16. Bernard S, Germi R, Lupo J, et al. Symptomatic cytomegalovirus gastrointestinal infection with positive quantitative real-time PCR findings in apparently immunocompetent patients: a case series. Clin Microbiol Infect 2015;21:1121.e1-e7.

17. Nikolich-Žugich J, van Lier RAW. Cytomegalovirus (CMV) research in immune senescence comes of age: overview of the 6th international workshop on CMV and immunosenescence. Geroscience 2017;39:245-249.

18. Al-Omari A, Aljamaan F, Alhazzani W, Salih S, Arabi Y.
Cytomegalovirus infection in immunocompetent critically ill adults: literature review. Ann Intensive Care 2016;6:110.

19. Frantzeskaki FG, Karampi ES, Kottaridi C, et al. Cytomegalovirus reactivation in a general, nonimmunosuppressed intensive care unit population: incidence, risk factors, associations with organ dysfunction, and inflammatory biomarkers. J Crit Care 2015; 30:276-281.

20. Ito K, Okuno T, Sawada A, et al. Recurrent aphthous stomatitis caused by cytomegalovirus, herpes simplex virus, and candida species in a kidney transplant recipient: a case report. Transplant Proc 2019;51:993-997. 